\title{
Incidence of Venous Thromboembolism and Mortality in Patients with Initial Presentation of COVID-19
}

\author{
Dimitrios Giannis ${ }^{1}$ - Matthew A. Barish ${ }^{2} \cdot$ Mark Goldin $^{1,2} \cdot$ Stuart L. Cohen ${ }^{1,3} \cdot$ Nina Kohn ${ }^{1} \cdot$ Eugenia Gianos ${ }^{3,4}$. \\ Saurav Chatterjee ${ }^{2,3} \cdot$ Martin Lesser $^{1,3} \cdot$ Kevin Coppa $^{5} \cdot$ Jamie S. Hirsch $^{1,3,5} \cdot$ Thomas McGinn $^{1,3}$. \\ Alex C. Spyropoulos ${ }^{1,3,6,7}$ ) on behalf of the COVID-19 Consortium Group
}

Accepted: 16 February 2021 / Published online: 5 March 2021

(C) The Author(s), under exclusive licence to Springer Science+Business Media, LLC part of Springer Nature 2021

\begin{abstract}
Venous thromboembolism (VTE) has emerged as an important issue in patients with COVID-19. The purpose of this study is to identify the incidence of VTE and mortality in COVID-19 patients initially presenting to a large health system. Our retrospective study included adult patients (excluding patients presenting with obstetric/gynecologic conditions) across a multihospital health system in the New York Metropolitan Region from March 1-April 27, 2020. VTE and mortality rates within $8 \mathrm{~h}$ of assessment were described. In 10,871 adults with COVID-19, 118 patients (1.09\%) were diagnosed with symptomatic VTE (101 pulmonary embolism, 17 deep vein thrombosis events) and 28 patients $(0.26 \%)$ died during initial assessment. Among these 146 patients, $64.4 \%$ were males, $56.8 \%$ were 60 years or older, $15.1 \%$ had a BMI > 35, and $11.6 \%$ were admitted to the intensive care unit. Comorbidities included hypertension (46.6\%), diabetes $(24.7 \%)$, hyperlipidemia (14.4\%), chronic lung disease (12.3\%), coronary artery disease (11.0\%), and prior VTE (7.5\%). Key medications included corticosteroids (22.6\%), statins (21.2\%), antiplatelets (20.6\%), and anticoagulants (20.6\%). Highest D-Dimer was greater than six times the upper limit of normal in 51.4\%. Statin and antiplatelet use were associated with decreased VTE or mortality (each $\mathrm{p}<0.01$ ). In COVID-19 patients who initially presented to a large multihospital health system, the overall symptomatic VTE and mortality rate was over $1.0 \%$. Statin and antiplatelet use were associated with decreased VTE or mortality. The potential benefits of antithrombotics in high risk COVID-19 patients during the pre-hospitalization period deserves study.
\end{abstract}

Keywords Venous thromboembolism · COVID-19 $\cdot$ Thrombosis $\cdot$ Outpatient

Alex C. Spyropoulos

aspyropoul@northwell.edu

1 Feinstein Institutes for Medical Research, Northwell Health, Manhasset, NY, USA

2 North Shore University Hospital, Northwell Health, Manhasset, NY, USA

3 Donald and Barbara Zucker School of Medicine at Hofstra/Northwell, Northwell Health, Hempstead, NY, USA

4 Division of Cardiology, Lenox Hill Hospital, Northwell Health, New York, NY, USA

5 Department of Information Services, Northwell Health, New Hyde Park, NY, USA

6 Zucker School of Medicine at Hofstra/Northwell, The Feinstein Institutes for Medical Research, 130 E 77th St, New York, NY 10075, USA

7 Department of Medicine, Anticoagulation and Clinical Thrombosis Services, Northwell Health at Lenox Hill Hospital, 130 E 77th St, New York, NY 10075, USA

\section{Highlights}

- COVID-19 has been associated with elevated rates of thromboembolic events in hospitalized patients.

- Antithrombotic guidance statements have disagreed on the need for primary thromboprophylaxis in outpatients with COVID-19, including those with thrombotic risk factors.

- In COVID-19 patients who initially presented to a large multihospital health system, the overall symptomatic VTE rate was $1.09 \%$ and the mortality rate was $0.26 \%$.

- The potential benefits of antithrombotics in COVID-19 patients during the pre-hospitalization period deserves further study. 


\section{Introduction}

The novel coronavirus disease 2019 (COVID-19) has been associated with elevated rates of thromboembolic events, while the pathophysiology underlying the formation of clots has not been fully elucidated yet $[1,2]$. The majority of thrombotic events represent venous thromboembolism (VTE) and involve both macro-vessel and micro-vessel disease and in situ fatal thrombosis [1,3].

The incidence of VTE events in hospitalized COVID-19 patients have been derived from retrospective studies, and most current data derived from large US studies have shown symptomatic VTE rates of $1.7 \%$ to $3.6 \%$ [4-6]. However, there is a lack of data regarding thromboembolic events in COVID-19 patients in the outpatient setting or with early presentation to the hospital, with one recently published study in pre-hospitalized patients with COVID-19 suggesting negligible rates of thrombosis [7]. Antithrombotic guidance statements have disagreed on the need for primary thromboprophylaxis in outpatients with COVID-19, including those with thrombotic risk factors [8,9]. Identifying and discriminating high-risk COVID-19 patients, especially in outpatient settings, remains a challenge [10].

Against this background, we investigated VTE and mortality rates in a large cohort of COVD-19 patients that presented initially within our multihospital health system in the New York metropolitan area during the height of the COVID-19 pandemic.

\section{Methods}

Our retrospective observational study included patients aged 18 years or older diagnosed with COVID-19 admitted to the Northwell Health multihospital system in the New York region between March 1-April 27, 2020. Patients admitted for obstetric/gynecologic reasons were excluded. We collected patient demographic characteristics, comorbidities, key medications administered at home or within $48 \mathrm{~h}$ if patients were admitted, vital signs, and main laboratory parameters. The study was performed with institutional review board (Northwell Health IRB) approval and waiver of informed consent. We only included patients that had a diagnosis of VTE or died within eight hours after presentation, which would have captured all events attributable to the outpatient setting (i.e., non hospital-acquired). The rationale for the combined primary outcome was that death is a competing endpoint for VTE; a large proportion of COVID-19 deaths may result from undiagnosed VTE [3]. Data was obtained from the enterprise inpatient electronic health record (EHR; Sunrise Clinical Manager, Allscripts, Chicago, IL).
VTE was defined as new acute deep vein thrombosis (DVT) or pulmonary embolism (PE) diagnosed by imaging performed by the Department of Radiology or by point-ofcare lower extremity ultrasound and manually verified by two attending radiologists. We identified major comorbidities by $I C D-10$ coding: hypertension, diabetes, hyperlipidemia, chronic lung disease (asthma and COPD), coronary artery disease, heart failure, chronic kidney disease/end stage renal disease, chronic liver disease, cancer, peripheral arterial disease/peripheral vascular disease; cerebrovascular disease, previous history of VTE, and smoking status.

Laboratory results included the first creatinine $(\mathrm{SCr})$, platelet (PLT), hemoglobin ( $\mathrm{Hb})$, hematocrit (Hct), alkaline phosphatase (ALP), alanine (ALT) and aspartate (AST) aminotransferase results within $48 \mathrm{~h}$ of admission. Maximum D-Dimer (Dd) was defined as the maximum value throughout the hospitalization for patients without VTE or maximum Dd prior to a VTE event for patients diagnosed with VTE. Dd was categorized as normal to less than 4 times the upper limit of normal (ULN), 4-6 times ULN, > 6 times ULN, and unknown. The ULN for Dd was $239 \mathrm{ng} / \mathrm{mL}$.

Baseline medications (administered at home or within $48 \mathrm{~h}$ after admission) included antiplatelets, corticosteroids, intravenous immunoglobulin (IVIG), biologic agents, rheumatologic anti-inflammatories, immunosuppressants, antivirals, angiotensin converting enzyme (ACE)/angiotensin receptor blockers (ARB), azithromycin, hydroxychloroquine (HCQ), chloroquine, antacids/antihistamines, famotidine, and statin. Anticoagulants were classified as "home" (prior to admission) or inpatient medications and were categorized as none, treatment dose, prophylactic dose, or unknown.

Continuous variables were reported as mean and standard deviation. Categorical variables were reported as number of events and percentage frequencies.

Subjects who were diagnosed with a VTE and/or expired within $8 \mathrm{~h}$ of start of hospital care were compared to subjects who did not meet these endpoints. The chi-square test was used to examine the association between each of antiplatelet use and statin use and VTE or death within $8 \mathrm{~h}$. For these comparisons, a Bonferroni adjustment was used, such that $\mathrm{p}<0.01$ was considered significant.

All analyses were performed with SAS version 9.4 (SAS institute, Cary North Carolina).

\section{Results}

The study population consisted of 10,871 adults diagnosed with COVID-19. Within the first eight hours of presentation, 28 patients $(0.26 \%)$ died and symptomatic VTE was diagnosed in 118 patients $(1.09 \%)$, of which 101 were PE $(0.93 \%)$ and $17(0.16 \%)$ were DVT events. Of the 118 patients with VTE, all stayed in the hospital longer than 
Table 1 Demographics and comorbidities of the population with VTE or death within $8 \mathrm{~h}$ post-admission

\begin{tabular}{|c|c|}
\hline & All \\
\hline All & $146(100 \%)$ \\
\hline \multicolumn{2}{|l|}{ Age } \\
\hline $18-59$ & $63(43.2 \%)$ \\
\hline $60-75$ & $39(26.7 \%)$ \\
\hline $75+$ & $44(30.1 \%)$ \\
\hline \multicolumn{2}{|l|}{ Gender } \\
\hline Female & $52(35.6 \%)$ \\
\hline Male & $94(64.4 \%)$ \\
\hline \multicolumn{2}{|l|}{ BMI } \\
\hline Unknown & $39(26.7 \%)$ \\
\hline$\leq 35$ & $85(58.2 \%)$ \\
\hline$>35$ & $22(15.1 \%)$ \\
\hline \multicolumn{2}{|l|}{ Race } \\
\hline Asian & $7(4.8 \%)$ \\
\hline Black & $45(30.8 \%)$ \\
\hline Other & $33(22.6 \%)$ \\
\hline Unknown & $7(4.8 \%)$ \\
\hline White & $54(37.0 \%)$ \\
\hline \multicolumn{2}{|l|}{ Ethnicity } \\
\hline Hispanic or Latino & $25(17.1 \%)$ \\
\hline Not Hispanic or Latino & $108(74.0 \%)$ \\
\hline Other/unknown & $13(8.9 \%)$ \\
\hline \multicolumn{2}{|l|}{ Past medical history } \\
\hline No cancer & $137(93.8 \%)$ \\
\hline Cancer & $9(6.2 \%)$ \\
\hline No hypertension & $78(53.4 \%)$ \\
\hline Hypertension & $68(46.6 \%)$ \\
\hline No CAD & $130(89.0 \%)$ \\
\hline CAD & $16(11.0 \%)$ \\
\hline No heart failure & $140(95.9 \%)$ \\
\hline Heart failure & $6(4.1 \%)$ \\
\hline No PAD or PVD & $139(95.2 \%)$ \\
\hline PAD or PVD & $7(4.8 \%)$ \\
\hline No/unknown VTE & $135(92.5 \%)$ \\
\hline VTE & $11(7.5 \%)$ \\
\hline No cerebrovascular disease & $140(95.9 \%)$ \\
\hline Cerebrovascular disease & $6(4.1 \%)$ \\
\hline No/unknown hyperlipidemia & $125(85.6 \%)$ \\
\hline Hyperlipidemia & $21(14.4 \%)$ \\
\hline No chronic liver disease & $142(97.3 \%)$ \\
\hline Chronic liver disease & $4(2.7 \%)$ \\
\hline No asthma & $132(90.4 \%)$ \\
\hline Asthma & $14(9.6 \%)$ \\
\hline No COPD & $140(95.9 \%)$ \\
\hline COPD & $6(4.1 \%)$ \\
\hline No diabetes & $110(75.3 \%)$ \\
\hline Diabetes & $36(24.7 \%)$ \\
\hline No ESRD or CKD & $142(97.3 \%)$ \\
\hline ESRD or CKD & $4(2.7 \%)$ \\
\hline
\end{tabular}

Table 1 (continued)

\begin{tabular}{ll}
\hline & All \\
\hline Active/former smoker & $20(13.7 \%)$ \\
Never smoker & $99(67.8 \%)$ \\
Unknown smoking history & $27(18.5 \%)$ \\
D Dimer Max & \\
Unknown & $56(38.3 \%)$ \\
Normal to $<4 \times$ ULN & $8(5.5 \%)$ \\
$4-6 \times$ ULN & $7(4.8 \%)$ \\
$>6 \times$ ULN & $75(51.4 \%)$ \\
ICU & \\
No & $118(80.8 \%)$ \\
Yes & $17(11.6 \%)$ \\
Unknown timing & $11(7.5 \%)$ \\
CCI & \\
0 & $12(8.2 \%)$ \\
$1-2$ & $46(31.5 \%)$ \\
$3-4$ & $30(20.6 \%)$ \\
$5+$ & $58(39.7 \%)$ \\
\hline
\end{tabular}

$B M I$ : Body mass index, $C A D$ Coronary artery disease, PAD Peripheral arterial disease, $P V D$ Peripheral vascular disease, VTE Venous thromboembolism, COPD Chronic obstructive pulmonary disease, ESRD End stage renal disease, CKD Chronic kidney disease, ULN Upper limit of normal, ICU Intensive care unit, $C C I$ Charlson comorbidity index

eight hours and 109 (92.4\%) patients remained alive at last follow-up.

Among the 146 patients who experienced symptomatic VTE or death, $64.4 \%(\mathrm{n}=94 / 146)$ were males, $56.8 \%$ $(\mathrm{n}=83 / 146)$ were 60 years or older, $15.1 \%(\mathrm{n}=22 / 146)$ had BMI $>35,13.7 \%(n=20 / 146)$ were active/former smokers, and $11.6 \%(n=17 / 146)$ were admitted to the intensive care unit (Table 1).

Main comorbidities included hypertension in $46.6 \%$ $(n=68 / 146)$, diabetes in $24.7 \%(n=36 / 146)$, hyperlipidemia in $14.4 \%(n=21 / 146)$, chronic lung disease in $12.3 \%$ $(n=18 / 146)$, coronary artery disease in $11.0 \%(n=16 / 146)$, and prior VTE in $7.5 \%(n=11 / 146)$ of patients. The Charlson Comorbidity Index (CCI) was greater than 2 in over $60 \%$ of the cohort. Key medications included hydroxychloroquine in $41.1 \%(\mathrm{n}=60 / 146)$, corticosteroids in $22.6 \%$ $(\mathrm{n}=33 / 146)$, statins in $21.2 \%(\mathrm{n}=31 / 146)$, antiplatelets in $20.6 \%(n=30 / 146)$, anticoagulants in $20.6 \%(n=30 / 146)$ with $14.4 \%$ on treatment dose and $6.2 \%$ on prophylactic dose, and azithromycin in $19.2 \%(n=28 / 146)$ (Table 2).

Mean $\mathrm{SpO} 2$ was $91.9 \% \pm 8.5 \%$, mean systolic blood pressure was $125.7 \pm 23.4 \mathrm{mmHg}$, mean diastolic pressure was $76.3 \pm 16.1 \mathrm{mmHg}$, mean heart rate was $105.4 \pm 24.1 \mathrm{bpm}$, mean respiratory rate was $24.0 \pm 8.9$ breaths per minute, and mean temperature was $37.1 \pm 0.9^{\circ} \mathrm{C}$. 
Table 2 Baseline treatment/medications of the population with VTE or death within $8 \mathrm{~h}$ post-admission

\begin{tabular}{|c|c|}
\hline Baseline treatment $/ \mathrm{n}$ & \\
\hline Hospital anticoagula & \\
\hline None & $145(99.3 \%)$ \\
\hline Prophylaxis dose & $1(0.7 \%)$ \\
\hline Treatment dose & $0(0.0 \%)$ \\
\hline Home Anticoagulati & \\
\hline Unknown & $50(34.3 \%)$ \\
\hline None & $66(45.2 \%)$ \\
\hline Prophylaxis dose & $9(6.2 \%)$ \\
\hline Treatment dose & $21(14.4 \%)$ \\
\hline Home or Hospital A & \\
\hline None & $68(46.6 \%)$ \\
\hline Present & $30(20.5 \%)$ \\
\hline NA & $48(32.9 \%)$ \\
\hline Steroids & \\
\hline None & $113(77.4 \%)$ \\
\hline Present & $33(22.6 \%)$ \\
\hline IVIG & \\
\hline None & $146(100.0 \%)$ \\
\hline Present & $0(0.0 \%)$ \\
\hline Biologic agents & \\
\hline None & $146(100.0 \%)$ \\
\hline Present & $0(0.0 \%)$ \\
\hline Rheumatologic anti- & \\
\hline None & $140(95.9 \%)$ \\
\hline Present & $6(4.1 \%)$ \\
\hline Immunosuppressant & \\
\hline None & $136(93.2 \%)$ \\
\hline Present & $10(6.8 \%)$ \\
\hline Antiviral medication & \\
\hline None & $145(99.3 \%)$ \\
\hline Present & $1(0.7 \%)$ \\
\hline ACE/ARB & \\
\hline None & $134(91.8 \%)$ \\
\hline Present & $12(8.2 \%)$ \\
\hline Azithromycin & \\
\hline None & $118(80.8 \%)$ \\
\hline Present & $28(19.2 \%)$ \\
\hline Hydroxychloroquine & \\
\hline None & $86(58.9 \%)$ \\
\hline Present & $60(41.1 \%)$ \\
\hline Chloroquine & \\
\hline None & $146(100.0 \%)$ \\
\hline Present & $0(0.0 \%)$ \\
\hline Famotidine & \\
\hline None & $129(88.4 \%)$ \\
\hline Present & $17(11.6 \%)$ \\
\hline Statin & \\
\hline None & $115(78.8 \%)$ \\
\hline Present & $31(21.2 \%)$ \\
\hline Antacid/antihistamir & \\
\hline None & $142(97.3 \%)$ \\
\hline Present & $4(2.7 \%)$ \\
\hline
\end{tabular}

$I V I G$ Intravenous immunoglobulin, $A C E$ Angiotensin-converting enzyme, $A R B$ Angiotensin II receptor blockers
Mean Hct was $40.4 \% \pm 5.5 \%$, mean $\mathrm{Hb}$ was $13.1 \pm 1.8 \mathrm{~g} /$ $\mathrm{dL}$, mean PLT was $286.7 \pm 118.1 \times 10^{9} / \mathrm{L}$, mean $\mathrm{SCr}$ was $1.39 \pm 1.06 \mathrm{mg} / \mathrm{dL}$, mean ALT was $54.1 \pm 53.6 \mathrm{U} / \mathrm{L}$, mean AST was $61.1 \pm 79.8 \mathrm{U} / \mathrm{L}$, and mean ALP was $106.3 \pm 96.0$ U/L. D-Dimer was $>6$ times ULN in $51.4 \%(n=75 / 146)$ of patients with VTE.

There was a significant association between statin use and antiplatelet use and decreased VTE or death $(0.86 \%$ versus $1.58 \%, \mathrm{p}<0.0021$ and $0.92 \%$ versus $1.31 \%, \mathrm{p}<0.0022$, respectively).

\section{Discussion}

Our analysis revealed an overall symptomatic VTE rate from mostly PE of $1.09 \%$ and a mortality rate of $0.26 \%$ in a large cohort of COVID-19 patients at initial presentation to a large multihospital system in the New York metropolitan area during the height of the pandemic. These events occurred in mostly older males with multiple comorbidities and deteriorated respiratory status and very elevated inflammatory markers, particularly D-dimer.

Our rates of symptomatic VTE and mortality during initial presentation are not negligible, and suggest a higher than expected thrombotic risk in pre-hospitalized patients with COVID-19, especially in those with known cardiovascular risk factors. The very high proportion of patients with PE (over 85\%) as a manifestation of VTE strongly suggest a process of local thromboinflammation of alveolar tissue in patients with severe COVID-19 pneumonia as a possible explanation of thrombosis [9]. Interestingly, Piazza et al. recently reported symptomatic VTE rates of $0.0 \%$ in COVID-19 outpatients [7]. Reasons for discrepancies between our data and those of Piazza et al. are unknown, but may reflect confounding and ascertainment and other bias.

Our population characteristics and findings are congruent with previous reports of high rates of early venous thromboembolism during the first days of hospitalization in older populations with an overall worse prognosis [11]. The overt elevation of D-Dimer $(>1,434 \mathrm{ng} / \mathrm{mL})$ in more than half our population diagnosed with VTE or deceased within the first eight hours further confirms the importance of this marker in the prognosis of COVID-19 patients.

Our study found a significant association between statin and antiplatelet use and an approximately $45 \%$ and $30 \%$ decrease in VTE or death, respectively. There are four ongoing placebo controlled trials examining primary thromboprophylaxis in high risk outpatients with COVID-19, including the large National Institute of Health trial [NCT04498273] and the large PREVENT-HD trial [NCT04508023] [12]. Our data suggest that there may be a subset of high risk outpatients with COVID-19, especially those with cardiovascular or thrombotic risk factors, 
including advanced age, elevated CCI, the presence of cardiopulmonary disease, and elevated D-Dimer, that may benefit from primary thromboprophylaxis. Whether this tendency for thrombosis plays an important role in COVID-19 disease progression requiring hospitalization and whether there is a role for prophylactic anticoagulation in the pre-hospital setting has yet to be established.

Our study has several strengths, including a large sample size of 10,871 patients allowing precise estimates of both VTE and mortality. The VTE events were systematically adjudicated and evaluated in a standardized way by experienced radiologists. A centralized COVID-19 hospitalization database, that was available soon after the pandemic struck our system, provided uniformity of both clinical and laboratory definitions. However, our study has several limitations. The true VTE rate may be underreported as VTE events may not have been confirmed by imaging studies due to concerns related to exposure of healthcare staff to COVID-19. In addition, non-systematic DVT screening may have resulted in underestimation of the true VTE incidence compared to previous studies that did employ these methods [13].

In conclusion, our study provides data regarding the symptomatic VTE and mortality rates of a large COVID-19 patient cohort during initial presentation to a multihospital system. Statin and antiplatelet use were associated with decreased VTE or mortality. These results indicate the need to study the potential benefits of antithrombotics in COVID19 patients during the pre-hospitalization period, especially in patients with cardiovascular or thrombotic risk factors.

\section{Disclosures}

SLC received funding support from the Association of University Radiologists GE Radiology Research Academy Fellowship (GERRAF) and Siemens Healthinners and was a consultant for Infervision 2019. ACS is a consultant for Boehringer Ingelheim, Janssen, Bayer, and Portola. DG, MAB, MG, NK, EG, SC, ML, KC, JSH, TM: None.

Acknowledgements We would like to acknowledge the COVID-19 Consortium Group at Northwell Health/Feinstein Institutes for Medical Research as well as the Broxmeyer Fellowship in Clinical Thrombosis.

Author Contributions ACS and DG contributed to the study design. All authors contributed to data analysis and interpretation, drafting the manuscript, and critical editing.

Funding The present work was partially funded by the Broxmeyer Fellowship in Clinical Thrombosis.

\section{References}

1. Spyropoulos AC, Weitz JI (2020) Hospitalized COVID-19 patients and venous thromboembolism: A perfect storm. Circulation 142(2):129-132. https://doi.org/10.1161/CIRCULATIO NAHA.120.048020

2. Giannis D, Ziogas IA, Gianni P (2020) Coagulation disorders in coronavirus infected patients: COVID-19, SARS-CoV-1, MERS-CoV and lessons from the past. J Clin Virol. https://doi. org/10.1016/j.jcv.2020.104362

3. Wichmann D, Sperhake J-P, Lütgehetmann M et al (2020) Autopsy findings and venous thromboembolism in patients with COVID19: a Prospective Cohort Study. Ann Intern Med 173(4):268-277. https://doi.org/10.7326/M20-2003

4. Hanif A, Khan S, Mantri N et al (2020) Thrombotic complications and anticoagulation in COVID-19 pneumonia: a New York City hospital experience. Ann Hematol 99(10):2323-2328. https://doi. org/10.1007/s00277-020-04216-x

5. Bilaloglu S, Aphinyanaphongs Y, Jones S, Iturrate E, Hochman J, Berger JS (2020) Thrombosis in hospitalized patients with COVID-19 in a New York City health system. JAMA 324:799801. https://doi.org/10.1001/jama.2020.13372

6. Tang N, Bai H, Chen X, Gong J, Li D, Sun Z (2020) Anticoagulant treatment is associated with decreased mortality in severe coronavirus disease 2019 patients with coagulopathy. J Thromb Haemost 18:1094-1099. https://doi.org/10.1111/jth.14817

7. Piazza G, Campia U, Hurwitz S et al (2020) Registry of arterial and venous thromboembolic complications in patients with COVID-19. J Am Coll Cardiol 76(18):2060-2072. https://doi. org/10.1016/j.jacc.2020.08.070

8. Gerotziafas GT, Catalano M, Colgan M-P et al (2020) Guidance for the management of patients with vascular disease or cardiovascular risk factors and COVID-19: position paper from VASEuropean Independent Foundation in Angiology/Vascular Medicine. Thromb Haemost. https://doi.org/10.1055/s-0040-1715798

9. Bikdeli B, Madhavan MV, Jimenez D et al (2020) COVID-19 and thrombotic or thromboembolic disease: implications for prevention, antithrombotic therapy, and follow-up. J Am Coll Cardiol. https://doi.org/10.1016/j.jacc.2020.04.031

10. Spyropoulos AC, Levy JH, Ageno W et al (2020) Scientific and Standardization Committee Communication: Clinical Guidance on the Diagnosis, Prevention and Treatment of Venous Thromboembolism in Hospitalized Patients with COVID-19. J Thromb Haemost 18(8):1859-1865. https://doi.org/10.1111/jth.14929

11. Le Jeune S, Suhl J, Benainous R et al (2020) High prevalence of early asymptomatic venous thromboembolism in anticoagulated COVID-19 patients hospitalized in general wards. J Thromb Thrombolysis. https://doi.org/10.1007/s11239-020-02246-w

12. Tritschler T, Mathieu M-E, Skeith L et al (2020) Anticoagulant interventions in hospitalized patients with COVID-19: A scoping review of randomized controlled trials and call for international collaboration. J Thromb Haemost 18(11):2958-2967. https://doi. org/10.1111/jth. 15094

13. Cui S, Chen S, Li X, Liu S, Wang F (2020) Prevalence of venous thromboembolism in patients with severe novel coronavirus pneumonia. J Thromb Haemost 18:1421-1424. https://doi.org/10.1111/ jth. 14830

Publisher's Note Springer Nature remains neutral with regard to jurisdictional claims in published maps and institutional affiliations. 